\title{
Validação de um instrumento de avaliação da satisfação dos discentes de cursos de graduação em Matemática
}

Validation of an instrument for evaluating the satisfaction of undergraduate students in Mathematics

\author{
V. B. R. Dutra; S. H. Ávila; V. L. D. Mattos \\ Instituto de Matemática, Estatística e Física, Universidade Federal do Rio Grande, Rio Grande-RS, Brasil \\ *nussa_rg@hotmail.com
}

(Recebido em 25 de abril de 2017; aceito em 22 de maio de 2017)

\begin{abstract}
O propósito desse artigo é descrever as etapas de validação de conteúdo e de validação de construto realizadas na construção de um instrumento que mensura o nível de satisfação dos estudantes dos cursos de Matemática Aplicada e Matemática Licenciatura de uma Instituição de Ensino Superior. Para efetivar este processo já foram desenvolvidas duas etapas: a primeira resultou na elaboração do instrumento preliminar, executada com base na revisão bibliográfica, e a outra, na sua primeira versão, resultante da validação de conteúdo, que se fundamentou na opinião de especialistas e de uma pequena amostra da população alvo: os estudantes da instituição. Atualmente, o estudo encontra-se na etapa de validação de construto, que se utiliza da análise fatorial. O escore total atribuído à satisfação será determinado por controladores fuzzy, usando a diferença entre a avaliação do item e sua importância.

Palavras-chave: validação de instrumento, avaliação da satisfação, curso de graduação em Matemática.
\end{abstract}

The purpose of this article is to describe the content validation and the construct validation used in the construct of an instrument that measures the level of satisfaction of undergraduate students in Applied Mathematics or Mathematics of un Undergraduate High School. To carry out this process, two stages have already been developed. The first one resulted in the elaboration of the preliminary instrument, based on the bibliographic review. The validation of the content, based on the opinion of the experts and a small sample of the target population: the students of the Institution, provided its first version. Currently, the study is in the step of the validation of the construct, which uses the factorial analysis. The total score attributed to the satisfaction will be determined by fuzzy controllers, using the difference between the evaluation of the item and its importance.

Keywords: Instrument validation, satisfaction assessment, degree course in Mathematics.

\section{INTRODUÇÃO}

No Ensino Superior, principalmente nos cursos relacionados à área de Ciências Exatas, como Engenharias e Matemática, são obtidos altos índices de reprovação e evasão já no primeiro semestre. Esses percalços acadêmicos, tanto a reprovação quanto a evasão, podem estar intrinsecamente ligados à satisfação do estudante. Este construto está relacionado com a qualidade dos serviços oferecidos pelas Instituições de Ensino Superior, ou seja, o acadêmico necessita usufruir de um ensino de qualidade, com professores qualificados profissionalmente, além de infraestruturas internas, externas e equipamentos adequados às suas necessidades. Se a qualidade percebida supera a expectativa, eles se sentem satisfeitos e, por consequência, se tornam mais motivados para obter um melhor desempenho acadêmico. Com base nesse problema, surgiu a motivação em desenvolver um instrumento para mensurar a satisfação dos acadêmicos dos cursos de Matemática Licenciatura e Matemática Aplicada da Universidade Federal do Rio Grande (FURG).

O processo de validação de uma escala se divide em pelo menos duas etapas: validação de conteúdo e validação de construto. A validação de conteúdo tem o objetivo de definir uma amostra de itens relevantes para os diferentes domínios que compõe o construto a ser mensurado: a satisfação. Segundo [9], a validade de conteúdo de um teste consiste em verificar se o teste constitui uma amostra representativa de um universo finito de comportamentos (domínio). É aplicável 
quando se pode delimitar a priori e com clareza um universo de comportamentos, como é o caso dos testes de desempenho, que pretendem cobrir um conteúdo delimitado por um curso específico.

A validade de construto consiste na utilização de ferramentas estatísticas para determinar a adequação do construto do instrumento de mensuração. Desta forma, objetiva-se comprovar se o conjunto de declarações, definido na validação de conteúdo, realmente reflete o construto a ser mensurado. Segundo Cooper (2003) [2], nesta etapa considera-se, além do instrumento de mensuração utilizado, a teoria. De acordo com os autores, tenta-se identificar os construtos implícitos que são mensurados, bem como determinar como os testes representam esses construtos. Assim, uma medida apresenta validade de construto no grau em que estiver de acordo com as correlações previstas em outras proposições teóricas. O método por meio do qual é definido operacionalmente o evento precisa corresponder a uma base teórica empírica. Depois de estabelecido que o construto é importante no sentido teórico, busca-se a adequação do instrumento. Se já houver uma medida do construto conhecida, os resultados obtidos são correlacionados com essa medida.

O objetivo deste artigo é descrever o processo de validação de conteúdo e de construto que está sendo realizado na construção do instrumento para medir satisfação discente, apresentando sua metodologia e alguns dos resultados obtidos.

\section{MATERIAL E MÉTODOS}

O estudo iniciou com uma extensa revisão de literatura sobre a construção de instrumentos desenvolvidos para medir a satisfação discente, sendo selecionados alguns textos. Uma análise crítica e reflexiva destes permite identificar as áreas a serem abordadas e os itens (declarações) a serem utilizados na construção de uma versão do instrumento, denominada de instrumento preliminar, a ser submetida à validação de conteúdo.

Participaram desta segunda etapa cinco especialistas de diferentes áreas: Matemática, Educação e Psicologia. Foi realizada uma reunião individual com cada especialista, solicitando a avaliação de cada área abordada e cada declaração proposta. Esses especialistas analisaram os seguintes quesitos: pertinência teórica, validade de aplicação e adequação ao construto.

Também participaram do processo de validação de conteúdo, estudantes de dois cursos de graduação em Matemática. A reunião com os estudantes destes cursos foi feita em duas etapas: a primeira envolveu doze estudantes de graduação em Matemática Licenciatura que, em conjunto, avaliaram o instrumento, enquanto a segunda foi realizada com quatro estudantes do curso de graduação em Matemática Aplicada, que analisaram o instrumento individualmente. Os dezesseis estudantes envolvidos realizaram a análise de cada item em relação a estruturação, apresentação, vocabulário, redação, forma de abordagem e, principalmente, em relação à clareza de linguagem.

As sugestões obtidas por especialistas e estudantes foram analisadas de forma reflexiva e crítica, sendo realizadas as alterações julgadas necessárias. Este procedimento permitiu elaborar a primeira versão do instrumento, encaminhada posteriormente para a validação de construto.

Essa segunda etapa do processo terá como população alvo os discentes dos cursos de Matemática Licenciatura e Matemática Aplicada de uma instituição de Ensino Superior Pública. O instrumento (apêndice) será aplicado nos alunos regularmente matriculados que se dispuserem a participar do estudo. A validade de construto será verificada por meio de uma Análise Fatorial (AF), caracterizada por ser um conjunto de técnicas estatísticas que permitem explicar o comportamento de um número relativamente grande de variáveis observáveis em termos de um número pequeno de fatores ou variáveis latentes [10].

O modelo da Análise Fatorial pode ser formulado expressando os escores atribuídos à importância do i-ésimo item como uma combinação linear, conforme Eq.1:

$$
\begin{gathered}
X_{1}-\mu_{1}=a_{11} \cdot F_{1}+a_{12} \cdot F_{2}+\cdots+a_{1 k} \cdot F_{k}+\varepsilon_{1} \\
X_{2}-\mu_{2}=a_{21} \cdot F_{1}+a_{22} \cdot F_{2}+\cdots+a_{2 k} \cdot F_{k}+\varepsilon_{2} \\
\quad \cdots \\
X_{n}-\mu_{n}=a_{n 1} \cdot F_{1}+a_{n 2} \cdot F_{2}+\cdots+a_{n k} \cdot F_{k}+\varepsilon_{n}
\end{gathered}
$$


onde:

$X_{i}$ é o escore atribuído à importância do $i$-ésimo item, sendo $i=1,2, \ldots, n$;

$F_{j}$ é a $j$-ésima variável latente, sendo $j=1,2,3, \ldots, k$;

$a_{i j}$ é a carga fatorial da $i$-ésima variável $\left(X_{i}\right)$ associada ao $j$-ésimo fator comum $\left(F_{j}\right)$;

$\mu_{i}$ é a parte do escore específica da $i$-ésima variável $\left(X_{\mathrm{i}}\right)$;

$\epsilon_{i}$ erro aleatório da i-ésima variável $\left(X_{i}\right)$.

Se for considerada a forma matricial, o modelo de AF é dado conforme Eq. 2.

$(\boldsymbol{X}-\mu)_{n \times 1}=\boldsymbol{A}_{n \times k} \cdot \boldsymbol{F}_{k \times 1}+\boldsymbol{\epsilon}_{n \times 1}$

A contribuição do i-ésimo item $\left(x_{i}\right)$ à mensuração da satisfação será dada a partir da diferença entre a pontuação atribuída à qualidade percebida do i-ésimo item $\left(Y_{i}\right)$ e a importância atribuída ao i-ésimo item $\left(X_{i}\right)$ conforme Eq.3:

$x_{i}=Y_{i}-X_{i}$

Finalizando, o escore total atribuído à satisfação do n-ésimo sujeito será determinado por controladores fuzzy, desenvolvido em três estágios: fuzzificação, inferência e defuzzificação. $\mathrm{O}$ universo de discurso é o intervalo [-100;100] e os termos linguísticos utilizados são: muito insatisfeito, insatisfeito, pouco satisfeito, satisfeito, bastante satisfeito e plenamente satisfeito, definidos pelos conjuntos fuzzy apresentados no Quadro 1 .

A base de regras será definida por especialistas, conforme o usual, enquanto o método de inferência será o de Mandani, o mais citado na literatura consultada. Para a defuzzificação, será utilizado o método do Centróide, definido conforme Eq.4.

$$
S_{n}=\frac{\sum_{l=1}^{L} \mu_{n l} \cdot s_{n l}}{\sum_{l=1}^{L} \mu_{n l}}
$$

onde:

$S_{n}$ é a satisfação associada ao j-ésimo fator do $n$-ésimo sujeito, $n=1,2, \ldots, N$;

$\mu_{n l}$ é uma área determinada a partir do grau de pertinência associada ao l-ésimo termo linguístico do n-ésimo sujeito.

Uma combinação linear entre os resultados encontrados para os fatores do n-ésimo sujeito fornecerá o escore representativo de sua satisfação.

\section{RESULTADOS E DISCUSSÃO}

Os estudos selecionados que embasaram inicialmente a construção do instrumento foram de Bonici e Araújo (2001) [1], Cunha et al. (2016) [3], Schleich et al. (2006) [11], Soares et al. (2002) [12] e Souza e Reinert (2010) [13]. Destes, Bonici e Araújo (2001) [1] e Souza e Reinert (2010) [13] contribuíram somente para a definição dos possíveis fatores a serem considerados na avaliação da satisfação. O estudo de Bonici e Araújo (2001) [1] considera que a satisfação discente deve ser mensurada em função de características do tutor (professor), aluno, disciplina, tecnologia, suporte e tutoria/professor. Já o estudo Souza e Reinert (2010) [13] considera características do corpo docente, estrutura curricular, características do curso, ambiente social, infraestrutura e questões pessoais.

A elaboração das declarações teve subsídios nos trabalhos Cunha et al. (2016) [3], Schleich et al. (2006) [11] e Soares et al. (2002) [12]. O Quadro 2 mostra a lista de declarações consideradas inicialmente, totalizando 35 , e os respectivos estudos que as propuseram. 
No instrumento preliminar, além dos dados de identificação, é solicitado ao estudante que atribua uma pontuação à importância da declaração e, posteriormente, à qualidade do desta no contexto onde está inserido.

Nas entrevistas com especialistas foi sugerido que as declarações fossem substituídas por itens, pois nesta forma poderiam influenciar a opinião dos respondentes. Também foi recomendado que as declarações 9, 13 e 19 deveriam ser agrupadas por categorias, pois facilitaria na avaliação. Evidenciaram que deveria ser evitado o uso de palavras repetidas na mesma frase, como na declaração 3. Além disso, sugeriram que as declarações $6,8,9,10,13,15,18,24$ fossem mais específicas, pois apresentavam um sentido muito amplo. Outra sugestão foi que as declarações com ideias duplas fossem evitadas, como em 3, 4 e 7. E, por último, consideraram que as declarações 21 e 22 possuíam o mesmo objetivo, sugerindo que uma delas fosse excluída.

Os estudantes do curso de graduação em Matemática Licenciatura consideraram a redação clara e sugeriram que os itens que tinham uma avaliação pessoal deveriam ser retirados, pois não dependiam de aspectos relativos à instituição ou ao curso. Logo, foram excluídas as declarações 15, 18, 21, 31, 32, 33, 34 e 35. Já os estudantes do curso de graduação em Matemática Aplicada sugeriram que os significados de alguns itens relacionados à educação fossem explicados, pois muitos desconhecem termos técnicos relacionados ao processo de ensino-aprendizagem. A declaração 24, que aborda sobre as metodologias diferenciadas em sala de aula, foi exemplificada para uma melhor compreensão dos estudantes que desconhecem esse termo.

A segunda versão do instrumento, resultante desta primeira análise está apresentada no Apêndice e foi composta por 27 itens que, de acordo com os autores, poderiam estar relacionados a três diferentes domínios: características da instituição (5 itens), recursos pedagógicos (4 itens) e características das disciplinas (18 itens). A determinação dos domínios e respectivos itens relevantes, entretanto, serão definidos na validação de construto.

\section{CONCLUSÃO}

O presente estudo descreve o processo de validação de conteúdo, uma etapa muito importante na construção de um instrumento por possibilitar identificar uma primeira amostra de itens relevantes para explicar os possíveis domínios de um construto. Sua execução, que contou com a colaboração de especialistas e estudantes de graduação em Matemática, conseguiu envolvê-los no processo e transcorreu de forma muito satisfatória.

O instrumento proposto, apresentado no Apêndice, foi considerado válido quanto ao seu conteúdo e aplicabilidade a uma população de estudantes de cursos de graduação em Matemática, objetivando mensurar sua satisfação. Salienta-se, entretanto, que ele ainda necessita passar por um processo de validação de construto e confiabilidade.

\section{AGRADECIMENTOS}

Agradecemos à CAPES pelo auxílio financeiro na forma de bolsa de mestrado Demanda Social, à Universidade Federal de Rio Grande e ao Programa de Modelagem Computacional desta instituição. 
Quadro 1 - Funções de pertinência associadas aos conjuntos fuzzy que definem os termos linguísticos

\begin{tabular}{|c|c|c|c|c|}
\hline \multirow{2}{*}{$\begin{array}{l}\text { Termo } \\
\text { linguístico } \\
\text { Muito } \\
\text { Insatisfeito }\end{array}$} & \multicolumn{4}{|c|}{ Função de pertinência } \\
\hline & $\mu_{M I}=$ & $\left\{\begin{array}{r}0 \\
1 \\
-50-x \\
20 \\
0\end{array}\right.$ & $\begin{array}{l}\text { se } \\
\text { se } \\
\text { se } \\
\text { se }\end{array}$ & $\begin{array}{l}x<-100 \\
-100 \leq x<-70 \\
-70 \leq x<-50 \\
x>-50\end{array}$ \\
\hline Insatisfeito & $\mu_{I}=$ & $\begin{array}{r}0 \\
\frac{x+70}{20} \\
1 \\
-10-x \\
40 \\
0\end{array}$ & $\begin{array}{l}\text { se } \\
\text { se } \\
\text { se } \\
\text { se } \\
\text { se }\end{array}$ & $\begin{array}{l}x<-70 \\
-70<x \leq-50 \\
x=-50 \\
-50 \leq x<-10 \\
x<-10\end{array}$ \\
\hline Pouco satisfeito & $\mu_{\mathrm{PS}}=$ & $\begin{array}{r}0 \\
\frac{x+50}{40} \\
1 \\
\frac{10-x}{20} \\
0\end{array}$ & $\begin{array}{l}\text { se } \\
\text { se } \\
\text { se } \\
\text { se } \\
\text { se }\end{array}$ & $\begin{array}{l}x<-50 \\
-50<x \leq-10 \\
x=-10 \\
-10 \leq x<10 \\
x>10\end{array}$ \\
\hline Satisfeito & $\mu_{S}=$ & $\begin{array}{c}0 \\
\frac{x+10}{20} \\
\frac{50-x}{40}\end{array}$ & $\begin{array}{l}\text { se } \\
\text { se } \\
\text { se } \\
\text { se } \\
\text { se }\end{array}$ & $\begin{array}{l}x<-10 \\
-10<x \leq 10 \\
x=10 \\
10 \leq x<50 \\
x<50\end{array}$ \\
\hline $\begin{array}{l}\text { Bastante } \\
\text { satisfeito }\end{array}$ & $\mu_{\mathrm{BS}}=$ & 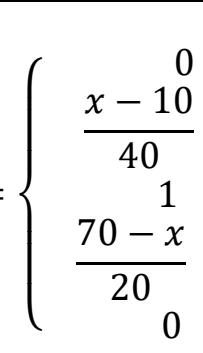 & $\begin{array}{l}\text { se } \\
\text { se } \\
\text { se } \\
\text { se } \\
\text { se }\end{array}$ & $\begin{array}{l}x<10 \\
10<x \leq 50 \\
x=50 \\
50 \leq x<70 \\
x<70\end{array}$ \\
\hline $\begin{array}{l}\text { Plenamente } \\
\text { satisfeito }\end{array}$ & $\mu_{P S}=$ & $\left\{\begin{array}{r}0 \\
x-50 \\
20 \\
1 \\
0\end{array}\right.$ & $\begin{array}{l}\text { se } \\
\text { se } \\
\text { se } \\
\text { se }\end{array}$ & $\begin{array}{l}x<50 \\
50 \leq x<70 \\
70 \leq x \leq 100 \\
x>100\end{array}$ \\
\hline
\end{tabular}


Quadro 2 - Declarações utilizadas no instrumento preliminar e instrumentos que as utilizaram

\begin{tabular}{|c|c|c|c|}
\hline Declarações & [3] & [11] & [12] \\
\hline $\begin{array}{l}\text { A infraestrutura física da Instituição (prédios, salas de aula, laboratórios, } \\
\text { ambientes de trabalho / estudo, auditórios) atende às necessidades. }\end{array}$ & & $\checkmark$ & \\
\hline $\begin{array}{l}\text { 2. A infraestrutura física das salas de aula (cadeiras, classes, equipamentos } \\
\text { de ventilação, paredes, pisos etc.) é adequada. }\end{array}$ & & $\checkmark$ & \\
\hline $\begin{array}{l}\text { 3. Os recursos e equipamentos audiovisuais disponíveis na Instituição (salas } \\
\text { de aula, laboratórios e auditórios) estão disponíveis e em bom estado, quando } \\
\text { existe necessidade de utilizá-los }\end{array}$ & $\checkmark$ & $\checkmark$ & \\
\hline $\begin{array}{l}\text { 4. Os equipamentos e softwares oferecidos nos laboratórios de informática } \\
\text { são adequados e de boa qualidade. }\end{array}$ & $\checkmark$ & $\checkmark$ & \\
\hline 5. O acervo disponível na biblioteca é atualizado e adequado à demanda. & & $\checkmark$ & \\
\hline 6. A segurança oferecida pela Instituição é adequada. & & $\checkmark$ & \\
\hline $\begin{array}{l}\text { 7. A equipe de limpeza mantém os ambientes em boas condições de higiene } \\
\text { e organização }\end{array}$ & & $\checkmark$ & \\
\hline $\begin{array}{l}\text { 8. O atendimento e a clareza das informações oferecidas pelos funcionários } \\
\text { das secretarias da Instituição são satisfatórios. }\end{array}$ & $\checkmark$ & $\checkmark$ & \\
\hline $\begin{array}{l}\text { 9. As referências indicadas nas disciplinas estão de acordo com os conteúdos } \\
\text { ministrados. }\end{array}$ & & & $\checkmark$ \\
\hline 10. A ementa das disciplinas está adequada a sua carga horária. & & & $\checkmark$ \\
\hline $\begin{array}{l}\text { 11. Os trabalhos/exercícios propostos são adequados aos conteúdos } \\
\text { desenvolvidos nas disciplinas? }\end{array}$ & & & $\checkmark$ \\
\hline 12. Os recursos pedagógicos são de boa qualidade. & & & $\checkmark$ \\
\hline 13. A sequência da grade curricular do Curso é adequada. & & & $\checkmark$ \\
\hline 14. A Instituição possui atividades extracurriculares diversificadas. & & $\checkmark$ & $\checkmark$ \\
\hline 15. O envolvimento pessoal em relação ao curso que frequenta é satisfatório. & & & $\checkmark$ \\
\hline 16. Existem eventos sociais realizados pela Instituição. & & & $\checkmark$ \\
\hline 17. A instituição possui programas e serviços de apoio financeiro ao estudante. & $\checkmark$ & & \\
\hline 18. A Instituição oferece condições de desenvolvimento pessoal do estudante. & $\checkmark$ & & \\
\hline 19. Os professores apresentam os conteúdos de forma clara. & & $\checkmark$ & \\
\hline 20. Os professores estão comprometidos a ensinar. & $\checkmark$ & & \\
\hline $\begin{array}{l}\text { 21. Os exemplos utilizados pelos professores auxiliam na compreensão do } \\
\text { conteúdo. }\end{array}$ & & & \\
\hline $\begin{array}{l}\text { 22. Os professores procuram relacionar a teoria com a prática profissional } \\
\text { durante as aulas. }\end{array}$ & & & \\
\hline 23. Os professores são qualificados profissionalmente. & & & $\checkmark$ \\
\hline $\begin{array}{l}\text { 24. Os professores já utilizaram algum tipo de metodologia diferenciada em } \\
\text { sala de aula. }\end{array}$ & & & $\checkmark$ \\
\hline $\begin{array}{l}\text { 25. Os professores abordam todos os conteúdos previstos nas ementas das } \\
\text { disciplinas. }\end{array}$ & & & $\checkmark$ \\
\hline $\begin{array}{l}\text { 26. Os professores relacionam os conteúdos com as demais disciplinas do } \\
\text { curso. }\end{array}$ & & $\checkmark$ & $\checkmark$ \\
\hline 27. Os professores incentivam os debates em sala de aula. & & & \\
\hline 28. Os professores são receptivos a novas ideias e diferentes pontos de vista. & & & $\checkmark$ \\
\hline 29. Os professores avaliam seus alunos com coerência. & & & $\checkmark$ \\
\hline $\begin{array}{l}\text { 30. Os professores disponibilizam horários para atendimentos individuais ou } \\
\text { extraclasse. }\end{array}$ & & & $\checkmark$ \\
\hline $\begin{array}{l}\text { 31. O estudante apresenta interesse em aprender os conteúdos desenvolvidos } \\
\text { durante às aulas }\end{array}$ & & & $\checkmark$ \\
\hline 32. O estudante permanece atento as aulas. & & & $\checkmark$ \\
\hline 33. Curso lhe desafia intelectualmente. & & & $\checkmark$ \\
\hline 34. Geralmente, os estudantes participam das discussões em sala de aula. & $\checkmark$ & $\checkmark$ & $\checkmark$ \\
\hline $\begin{array}{l}\text { 35. Os estudantes têm oportunidades de realizarem perguntas sobre o } \\
\text { conteúdo abordado em sala de aula. }\end{array}$ & & $\checkmark$ & $\checkmark$ \\
\hline
\end{tabular}




\section{REFERÊNCIAS BIBLIOGRÁFICAS}

1. Bonici RMC, Araújo JC. Medindo a satisfação dos estudantes em relação a disciplina on-line de Probabilidade e Estatística. 2011. Disponível em: 〈http://www.abed.org.br/congresso2011/cd/190.pdf〉. Acesso em: 12 jan 2016.

2. Cooper DR, Schindler PS. Métodos de Pesquisa em Administração. 7ª ed. Porto Alegre: Bookman, 2003. 640p.

3. Cunha PR, Gomes G, Beck F. Satisfação dos estudantes do curso de Ciências Contábeis: estudo em Universidades Públicas de Santa Catarina. Contabilidade Vista \& Revista. 2016; 27(1):42-62.

4. Davis JC, Swanson S. Navigating satisfactory and dissatisfactory classroom incidents. J Education Business. 2001; 76(5):245-250.

5. Ferreira A. Uma Análise das Dimensões da Qualidade de Serviços em Educação Superior para Estabelecimento de Cursos de Pós-Graduação [dissertação]. Porto Alegre (RS): Universidade Federal do Rio Grande do Sul, 2005. 142 p.

6. Martins FA. Satisfação acadêmica: construção de uma escala. In: IV Anais do IV Congresso galaicoportuguês de Psicopedagogia; 1998 Set 24-26; Braga, Portugal: Universidade do Minho, p.188-193.

7. Martins GDA. Sobre confiabilidade e validade. Revista Brasileira de Gestão de Negócios. 2006; 8(20): $1-12$.

8. Neves AB, Ramos CF. A Imagem das Instituições de Ensino Superior e a Qualidade do Ensino de Graduação: a percepção dos acadêmicos do curso de administração. Revista de Economia e Administração. 2002; 1(1):75-86.

9. Pascarella ET, Terenzini PT. How College Affects Students: a third decade of research. $2^{\mathrm{a}}$ ed. San Francisco: Jossey-Bass. 2005. 815 p.

10. Pasquali L. Análise Fatorial para pesquisadores. Brasília: LabPAM, 2005. 301 p.

11. Schleich ALR, Polydoro SAJ, Santos AAA. Escala de satisfação com a experiência acadêmica de estudantes do Ensino Superior. Aval Psicológica. 2006; 5(1):11-20.

12. Soares APC, Vasconcelos RM, Almeida LS. Adaptação e satisfação na Universidade: apresentação e validação do questionário de satisfação acadêmica. Contextos e dinâmica da vida acadêmica, Guimarães: Universidade do Minho, 2002, 153-165.

13. Souza AS, Reinert JN. Avaliação de um curso de ensino superior através da satisfação/insatisfação discente. Avaliação: Revista da Avaliação da Educação Superior. 2010; 15(1):159-176.

14. Veiga RT, Alves JED, Moura AI, Deister ALC. Adquirindo uma visão mais abrangente da qualidade do ensino de administração. In: VII Ensino da administração: estado da arte da avaliação. Anais do VII ENANGRAD; 1996 Out 23-26; Florianópolis, SC, Brasil: ANGRAD. 
APÊNDICE - Versão do instrumento resultante da validação de conteúdo

Parte 1 - Dados de Identificação

1) Idade: anos

2) Gênero: ( ) feminino

( ) masculino

3) Estado Civil: ( ) solteiro (a)

( ) casado (a)

( ) separado(a)

( ) viúvo (a)

4) Você trabalha? ( ) sim ( ) não

Caso "sim", informe o turno: ( ) manhã

( ) tarde ( ) noite

5) Qual a quantidade de pessoas que compõe sua família?

6) Qual a renda familiar, ou seja, a soma da renda de todas as pessoas que moram com você?

( ) até 1 salário mínimo;

( ) de 1,1 a 3 salários mínimos;

( ) de 3,1 a 7 salários mínimos;

( ) mais de 7 salários mínimos;

( ) não gostaria de informar.

7) Curso que frequenta: ( ) Matemática Aplicada ( ) Matemática Licenciatura

8) Encontra-se padrão no curso? ( ) sim não

9) Qual a quantidade de disciplinas já cursadas e concluídas com aprovação?

10) Possui algum tipo de bolsa ou auxílio financeiro? ( ) sim

( ) não

Caso "sim", especifique o tipo:

11) Participa de atividades extracurriculares (eventos científicos, atividades de pesquisa, monitoria, …..)? ( ) sim ( ) não

12) Qual foi o ano de conclusão do Ensino Médio?

13) Qual a modalidade de ensino cursada durante o Ensino Médio?

( ) Ensino Médio Tradicional

( ) Ensino Médio Profissionalizante ( técnico)

( ) Ensino Médio Profissionalizante (magistério)

( ) EJA

( ) Outra modalidade

Em caso de "outra modalidade", especifique o tipo

14) Tipo de escola em que cursou o Ensino Médio: ( ) Público ( ) Privado ( ) Ambos 
Parte II - Avalie os itens apresentados abaixo, atribuindo duas pontuações entre zero (ruím) e 100 (excelente). A primeira deve representar a importância do item para a sua satisfação em relação ao curso de graduação. A segunda deve representar a qualidade percebida em relação ao serviço oferecido pela instituição em que você está inserido.

\begin{tabular}{|c|c|c|}
\hline Itens & Imp. & Qual. \\
\hline $\begin{array}{l}\text { 1. Infraestrutura física da Instituição (prédios, salas de aula, laboratórios, ambientes de } \\
\text { trabalho/estudo, auditórios). }\end{array}$ & & \\
\hline $\begin{array}{l}\text { 2. Infraestrutura física das salas de aula (cadeiras, classes, equipamentos de ventilação, } \\
\text { paredes, pisos etc.). }\end{array}$ & & \\
\hline $\begin{array}{l}\text { 3. Recursos e equipamentos audiovisuais disponíveis na Instituição (salas de aula, } \\
\text { laboratórios e auditórios). }\end{array}$ & & \\
\hline 4. Equipamentos e softwares oferecidos nos laboratórios de informática. & & \\
\hline 5. Acervo disponível na biblioteca. & & \\
\hline 6. Segurança (vigias, porteiros, iluminação...) oferecida pela Instituição. & & \\
\hline 7. Limpeza dos ambientes. & & \\
\hline 8. Informações oferecidas pelos funcionários das secretarias da Instituição. & & \\
\hline $\begin{array}{l}\text { 9. Concordância das referências bibliográficas com os conteúdos ministrados nas } \\
\text { disciplinas. }\end{array}$ & & \\
\hline 10. Ementa das disciplinas (ou conteúdo programático) adequada a sua carga horária. & & \\
\hline $\begin{array}{l}\text { 11. Adequação entre atividades (trabalhos, exercícios, ...) e conteúdos desenvolvidos nas } \\
\text { disciplinas. }\end{array}$ & & \\
\hline $\begin{array}{l}\text { 12. Recursos pedagógicos (atividades que auxiliem a aprendizagem, como jogos, } \\
\text { desafios, utilização de máquinas/equipamentos,....). }\end{array}$ & & \\
\hline 13. Grade curricular. & & \\
\hline 14. Atividades extracurriculares diversificadas. & & \\
\hline $\begin{array}{l}\text { 15. Envolvimento pessoal em relação ao curso (frequência, cumprimento de horários, } \\
\text { participação em atividades não obrigatórias,.....). }\end{array}$ & & \\
\hline $\begin{array}{l}\text { 16. Eventos sociais que promovam confraternização entre os estudantes realizados pela } \\
\text { Instituição. }\end{array}$ & & \\
\hline 17. Programas e serviços de apoio financeiro ao estudante oferecidos pela Instituição. & & \\
\hline 18. Clareza por parte dos professores nas apresentações dos conteúdos. & & \\
\hline 19. Comprometimento com o ensino. & & \\
\hline 20. Relação entre e a teoria e a prática profissional durante as aulas. & & \\
\hline 21. Qualificação profissional dos docentes. & & \\
\hline 22. Cumprimento dos conteúdos previstos nas ementas das disciplinas. & & \\
\hline 23. Interdisciplinaridade entre as disciplinas do curso. & & \\
\hline 24. Incentivo a debates em sala de aula. & & \\
\hline 25. Receptividade dos professores em relação a novas ideias e diferentes pontos de vista. & & \\
\hline 26. Coerência nas avaliações das disciplinas. & & \\
\hline 27. Atendimento extraclasse (professores/monitores). & & \\
\hline
\end{tabular}

Responda: 1) O que fez você escolher a FURG para cursar seu curso de graduação?

2) Por que você escolheu esse curso de graduação? 